\title{
Importance of Autoinfection to the Epidemiology of Polycyclic Foliar Disease
}

\author{
Christopher C. Mundt
}

Department of Botany \& Plant Pathology, 2082 Cordley Hall, Oregon State University, Corvallis, OR 97331-2902.

Accepted for publication 8 June 2009.

\begin{abstract}
Mundt, C. C. 2009. Importance of autoinfection to the epidemiology of polycyclic foliar disease. Phytopathology 99:1116-1120.

Autoinfection (infection resulting from inoculum produced on the same host unit) can result in strongly clustered disease at the local scale. In contrast, much epidemiological theory incorporates the simplification of spatially random or uniform infection. Earlier studies suggested only low to moderate levels of autoinfection, especially when the host unit is small. However, several studies published within the last 5 years suggest

indicated. I discuss the potential importance of accounting for high autoinfection rates in example epidemiological processes that occur at different spatial scales: microbial interactions on the phylloplane, temporal disease progression in plant populations, and spatiotemporal disease spread at the landscape scale. Accounting for high autoinfection rates can have important qualitative and quantitative consequences for epidemiological processes, and further studies of autoinfection will contribute significantly to our understanding of epidemics.
\end{abstract} that autoinfection rates may be substantially higher than previously
Additional keywords: dispersal, growth models, spatiotemporal spread.
Knowledge of pathogen dispersal patterns is crucial to a better understanding of the local increase of disease and its subsequent spread. An important aspect in this regard is the concept of autoinfection versus alloinfection. These terms originally were proposed by Robinson (45), who considered autoinfection to be "infection in which the donor (infector) host individual is the same as the receptor (infected) host individual." Conversely, alloinfection was considered to be "infection in which the donor (or infector) host is a different individual from the recipient (or infected) host individual." The concept has since been extended to both larger and smaller spatial scales. For example, in studying mosaics of host genotypes, autoinfection was considered to be infection resulting from propagules produced from the same unit of contiguous plants of the same genotype, while alloinfection was considered to be infections resulting from propagules produced on other genotype units in the population $(35,49,58)$. Towards the other end of the spatial scale, autoinfection has been measured in the field (24) and varied in models (56) when the host unit was that of an individual leaf, or even subsections of a single leaf.

For purposes of this letter, I consider autoinfections to be infections arising from inoculum produced on the same host unit and alloinfections to be infections arising from inoculum produced on other host units. Conceptually, a plant is the most intuitive host unit, but a subunit of a plant or a group of contiguous plants may be more appropriate for other applications. Both autoinfections and alloinfections commonly occur simultaneously on the same host unit, of course. For some purposes, the relative numbers of autoinfections and alloinfections may be of most interest, and can be expressed as a proportion of total infections or as a ratio of autoinfections to alloinfections. The relative number of autoinfections and alloinfections will often change during the course of an epidemic. Autoinfection and alloinfection

Corresponding author: C. C. Mundt; E-mail address: mundtc@ science.oregonstate.edu

doi:10.1094/PHYTO-99-10-1116

(c) 2009 The American Phytopathological Society rates can also be designated as numbers of infections that arise per unit time.

The importance of autoinfection to disease epidemiology and pathogen evolution in mixtures of host genotypes has been given some emphasis, especially in models $(12,33,50)$. Autoinfection has received very little attention beyond that area of study however. For example, autoinfection and alloinfection have not been studied sufficiently to merit inclusion among over 1,000 topics indexed in The Study of Plant Disease Epidemics (29), despite the fact that this text is a remarkably thorough treatment of plant disease epidemiology. Similarly, the three references to autoinfection or alloinfection in the second edition of The Epidemiology of Plant Diseases (6) are all with respect to disease dynamics in host mixtures.

Below I briefly discuss approaches used to measure or estimate autoinfection and alloinfection, including recent evidence indicating that autoinfection may be substantially greater than previously suggested. I will then discuss example epidemiological processes for which high autoinfection rates could be of substantial importance. I restrict discussion to pathogens that are polycyclic and aerially transmitted.

\section{ESTIMATING AUTOINFECTION AND ALLOINFECTION}

From an operational standpoint, estimating autoinfection and alloinfection involves determining the number of infections that derive from inoculum produced on the same host unit compared with infections that derive from inoculum produced on other host units. Direct measurements have rarely been done, however (24, 55). In many cases, infections were either not measured on the source plants, or else the source plants were grown differently (e.g., transplanted or grown in pots) as compared to the remainder of the field (24). In absence of reliable data from the source plants, one can extrapolate dispersal models back to the source, but this procedure can be very inaccurate and is generally not recommended (36). One can also use spatially explicit simulation models to determine the number of autoinfections and alloinfections that occur during the course of an epidemic (37). The 
accuracy of such simulations, however, depends critically on dispersal functions that can be validated and parameterized from appropriate field data.

Measuring autoinfection directly can be technically quite difficult and may require counting large numbers of infections on single leaves (36). There also are sources of potential bias. For example, physical handling and high inoculation rates may stress source plants, resulting in unrepresentative measures of autoinfection $(3,24)$. Source plants often are inoculated heavily to ensure that there is a sufficient number of alloinfections to count. As discussed in more detail below, Lannou et al. (24) have recently shown that host saturation caused by high inoculation levels may result in substantial underestimation of autoinfection that would result from a single infection. In addition, the highly variable nature of dispersal and of infection may require a method to account for effects of overdispersion on parameter estimates (24).

Measuring alloinfection also presents technical difficulties, as it requires accounting for all infections that could occur at all possible distances from the source. Wind-dispersed pathogens often have "fat-tailed" dispersal gradients (dispersal kernels) that may extend to long distances $(14,15)$. Though propagule numbers often drop off very rapidly when measured along a transect $(14,29)$, the total host area increases with the square of distance. If the host population extends over large areas, the overlapping of dispersal tails among host units may contribute substantially to the total number of alloinfections that occur $(32,34)$. Thus, an accurate estimate of alloinfection may require either counts of very small numbers of infections in very large areas or else sufficiently large sources of inoculum to produce a detectable number of infections per unit area at far distances. Neither of these two approaches is practical experimentally in most cases.

Attempts have been made to estimate autoinfection and alloinfection by inoculating individual plants (or small groups of plants), allowing primary infection to pass through a single latent period, and then measuring the resulting secondary infections on the source plant(s) and other plants in the population. Integrating over all plants in the population, other than the source, provides a measure of alloinfection. Using this approach, autoinfection was estimated to be 5 to $10 \%$ for oat (Avena sativa) stem rust caused by Puccinia graminis f. sp. avenae $(26,27)$ and $13 \%$ for oat crown rust caused by $P$. coronata $(36,37)$.

In other cases, intrinsic rates of disease increase (apparent infection rates sensu Vanderplank [53]) have been measured on individual host units in comparison to populations of host units. In controlled environment studies using 5-cm-diameter host units of barley (Hordeum vulgare), intrinsic growth rates of powdery mildew (caused by Blumeria graminis f. sp. hordei) at the population level ranged from $r=0.39$ to 0.75 /day depending on host density. Extrapolation of the rate-density regressions to the $y$-intercept provided an estimate of $r=0.25$ for a single host unit, i.e., autoinfection, while direct measurement on individual units in a separate experiment yielded an estimate of $r=0.12$ (5). Similarly, Fried et al. (11) calculated a mean intrinsic growth rate of $0.0952 /$ day for powdery mildew (caused by B. graminis f. sp. tritici) on individual wheat (Triticum aestivum) plants grown between rows of barley. In comparison, whole-plot infection rates in an experiment conducted in the same year ranged from 0.14 to 0.21 . Because the basic infection rate (number of progeny lesions produced per parent lesion per day of the infectious period) decreases exponentially with a linear decrease of the apparent infection rate (53), these data also suggest low autoinfection rates. In both of these studies, however, growth rates on individual host units were measured in different environments than that of the population-level estimate, thus confounding the comparisons of autoinfection versus alloinfection.

Newer tools such as genetically marked strains and quantitative PCR would seem to hold potential for more accurately dis- tinguishing among autoinfections, alloinfections, and background contamination from outside sources. To my knowledge, however, such tools have not yet been used in studies of autoinfection and alloinfection.

\section{MORE RECENT APPROACHES TO ESTIMATING AUTOINFECTION AND ALLOINFECTION}

Several studies published within the last 5 years suggest that autoinfection may be considerably higher than indicated above. In a set of meticulous studies, Lannou et al. (24) showed that local host saturation greatly reduces the number of autoinfections when infection levels are high, thus masking the potential importance of autoinfection that occurs early in an epidemic. They measured autoinfection of wheat leaf rust (caused by Puccinia triticina) by identifying isolated wheat leaves containing single, naturally occurring pustules, and then determining the number of new infections that occurred on each of those leaves one generation later. Mean values of autoinfection for single pustules ranged from 47 to 199/leaf/generation over the 4-year study.

Lannou et al. (24) also recorded infection patterns showing a very strong clustering of autoinfections in "microsites" around parent pustules. This pattern would suggest a large saturation effect when leaves contain multiple parent infections per leaf. To quantify this relationship, Lannou et al. (24) measured autoinfection on naturally and artificially inoculated leaves with a range of number of parent infections. They found that an increasing number of parent pustules/leaf greatly reduced the reproductive rate of the pathogen, and could be described by the equation

$$
y=\mu x^{\alpha}
$$

where $y$ is the number of progeny lesions, $\mu$ is the autoinfection rate per parent lesion in absence of host saturation, $x$ is the number of parent lesions, and $\alpha$ accounts for the degree of host saturation. When $\alpha$ is $<1$, negative density dependence accounts for saturation of potential infection sites. Lannou et al. (24) estimated $\alpha$ to be 0.41 in their wheat leaf rust studies. If $\alpha=0.41$, autoinfection of the pathogen would be reduced by 33, 60, and $75 \%$ with only 2,5 , and 10 initial pustules/leaf, respectively. Given that the carrying capacity for wheat leaf rust is on the order of hundreds per leaf, even low percentages of disease attained by artificial inoculation could mask the autoinfection capacity of a pathogen that can occur from isolated pustules early in an epidemic. Because autoinfections result from locally produced inoculum, they will be substantially more impacted by local host saturation than are alloinfections, which result from propagules that arrive more randomly with respect to the location of previous infections.

High autoinfection rates also were reported for stem rust of ryegrass (Lolium perenne), caused by Puccinia graminis subsp. graminicola. In this case, the pathogen was found to sporulate on the inside surface of inoculated flag leaf sheaths, resulting in infection of emerging stems and inflorescences (42). Autoinfection via this mechanism can cause greater than $50 \%$ of the total stem rust infection in a perennial ryegrass crop (43); additional autoinfections will certainly occur on exposed plant parts via aerial transmission. Infection of stems enclosed within leaf sheaths could be a general phenomenon for stem rusts of grass species, including wheat stem rust (42). Further, earlier season sheath infection by rusts and other pathogens could potentially result in infections of emerging leaves, as well as stems and inflorescences.

Vereijssen et al. (55) studied the importance of autoinfection by evaluating extensive field data for Cercospora beticola, the causal agent of Cercospora leaf spot of sugarbeet (Beta vulgaris). They used a set of linked differential equations to partition the total amount of disease on a target leaf layer of a plant into that 
contributed by that same layer, by layers above and below the target, and by neighboring plants within rows. In an analysis pooled over eight experiments, autoinfection contributed five times more to disease increase than did alloinfections, and alloinfections were calculated to have made no contribution at all to disease increase in one of the experiments. It should be noted, however, that $C$. beticola is a splash-dispersed pathogen, which would be expected to have higher autoinfection rates than wind-disseminated pathogens owing to steeper dispersal gradients $(12,33)$.

Willocquet and Savary (56) used a simulation model to study epidemic progression at three spatial scales: within leaves, among leaves on the same plant, and among plants. They then incorporated into their model data on spatial aggregation from field studies of a range of pathogens with differing modes of transmission, which were also collected at different spatial scales. Autoinfection slowed progression of the simulated epidemics to a much greater degree when data were available to model spatial aggregation within plants, as opposed to when data were available only at the plant or plant population levels (note, however, that autoinfection may not always slow disease progression [59, spatiotemporal spread in the landscape section of this paper]). They concluded that autoinfection may play a larger role in field epidemics than was initially suggested by simulations that disregarded small-scale disease aggregation. This conclusion is consistent with the field results of Lannou et al. (24), which suggest that it may be critically important to consider aggregation of disease at small spatial scales. In contrast, most plant disease data are collected at the level of individual plants or populations of plants.

It thus appears that autoinfection can be a highly significant contributor to plant disease epidemics, even when the host unit in question is very small, e.g., a single plant of a grass species. More confirmatory evidence is needed, including for a wider range of host/pathogen combinations. The motivation for obtaining such information depends, in part, on the importance of autoinfection to epidemiological processes. Below, I briefly discuss a few examples of epidemiological processes that could be significantly impacted by high autoinfection rates.

\section{EPIDEMIOLOGICAL IMPLICATIONS}

Pathogen competition on the phylloplane. Competition can occur on the phylloplane between pathogen genotypes $(20,39)$, pathogen species $(1,2,8)$, and between pathogens and microbial antagonists (21). Experimentally, it is common to apply competing organisms to the leaf surface as uniformly as possible. If autoinfection is important, however, one might expect colonies to be much more clustered in natural epidemics, potentially reducing the degree of competition between organisms (57). Such spatial clustering also could greatly reduce the effectiveness of inundative biological control by resulting in sites inaccessible to microbial antagonists (19).
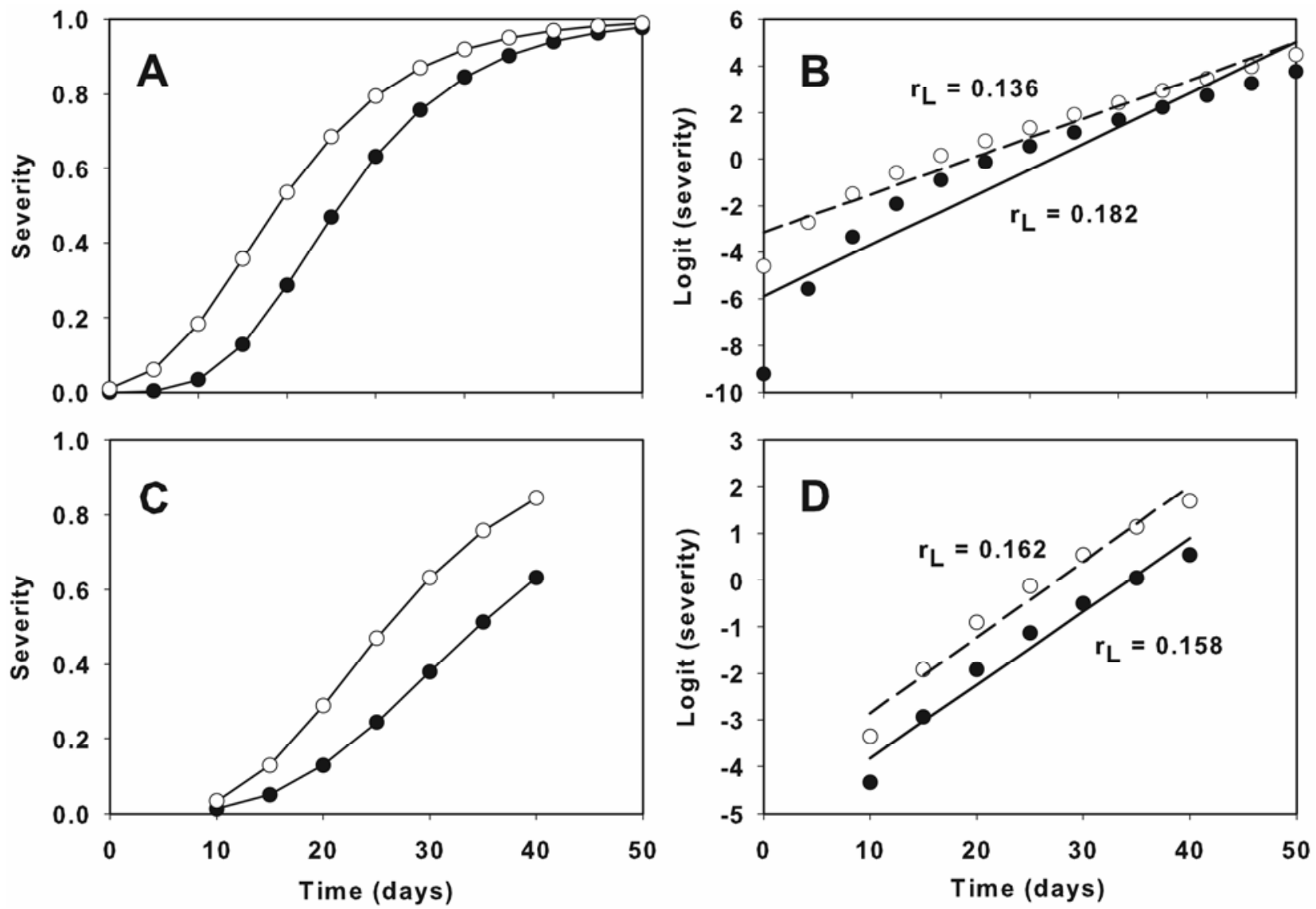

Fig. 1. Fitting the logistic growth equation to disease progression data with a derivative $(d y / d t)$ that is skewed to the right when plotted against time can result in significant bias of rate estimates. A, Disease progress curves produced from the Gompertz model (29) with a rate parameter of 0.1/day and initial disease severity of $0.01 \%$ (filled circles) or $1.0 \%$ (open circles). B, Application of the logistic equation to disease progress curves shown in $\mathbf{A}$ result in a logistic growth rate ( $r_{\mathrm{L}}$ ) for the epidemic beginning from 1.0\% initial disease (open circle) that is about $25 \%$ less than for the epidemic beginning from $0.01 \%$ initial disease severity (closed circles), despite the fact that the progress curves were derived from the Gompertz model with identical growth rates. C, Disease progress curves produced from the Gompertz model (29) with an initial disease severity of $0.01 \%$ and growth rates of $0.075 /$ day (filled circles) or $0.1 /$ day (open circles). Disease progression data are "censored", as data are first recorded 10 days after appearance of initial disease and the epidemic does not attain 100\% severity by the end of the growing season. D, Application of the logistic equation to disease progress curves shown in $\mathbf{C}$ result in logistic growth rates $\left(r_{\mathrm{L}}\right)$ that are highly similar despite the fact that one of the progress curves resulted from the Gompertz model with a rate $(0.075 /$ day, closed circles) that was $25 \%$ less than that of the other progress curve (0.1/day, open circles). 
Temporal disease progress in plant populations. The logistic growth equation is commonly fit to disease progression data from experimental plots and farmers' fields (53), usually by regressing the logit of disease proportion $[\ln (y /(1-y))]$ against time, where $y$ is disease proportion (29). The slope of the regression is an estimate of the infection rate, $r$. The logistic equation assumes a $1: 1$, negative correspondence between the amount of visible disease present and the number of remaining infection sites. If autoinfection results in localized host saturation (e.g., equation 1), then available infection sites would be expected to saturate more quickly than predicted by the logistic equation, resulting in a derivative $(d y / d t)$ that is skewed to the right when plotted versus time $(t)$ and a nonrandom pattern of residuals when fitting the logistic equation to such data. Indeed, it is very common for such a nonrandom pattern of residuals to result when fitting the logistic model to field data, with logit-transformed data increasing very rapidly at low disease levels, e.g., less than 5\% (4). One result of this pattern is a negative relationship between initial disease level and the infection rate as measured by the logistic growth equation (Fig. 1A and B). In fact, three studies $(13,44,46)$ incorporating a combination of observational and experimental data reported a negative association between the infection rate estimated by fitting the logistic equation and the level of initial disease severity measured in the field for five different host/pathogen systems. Similar problems can result, when data for disease progress curves with derivatives that are skewed to the right are incomplete ("censored"), as is very commonly the case for field data. Fitting the logistic equation to such data can bias estimated rates upward for the slower epidemics relative to faster epidemics, thus masking the true rate effects (Fig. 1B and D; C. C. Mundt, unpublished data). In addition, the logistic equation can significantly overestimate the epidemiological impacts of sanitation if the true underlying model is skewed (4).

There are factors in addition to autoinfection that could result in disease progress curves that are skewed to the right, e.g., the influence of latent infections on host carrying capacity (10), density-dependent pathogen reproduction (17), and bias in visual disease estimates (41). Distinguishing the relative contribution of autoinfection and other potential mechanisms to asymmetry of disease progress curves is an important research topic itself.

Spatiotemporal spread in the landscape. The spatiotemporal spread of plant disease from an initial focus has been described as a wavefront with a velocity that is either constant $(31,52)$ or increasing $(7,9,38)$, and may apply from the field to the continental scale $(16,38,48)$. For nearly all such models, however, there is an assumption that the local intensification of disease is independent of inoculum produced from other locations (29). If current models are considered to be realistic representations of spatiotemporal spread, then local build-up (which can be considered autoinfection at the landscape scale) must dominate over migration of inoculum from other parts of the host population (alloinfection). There is some evidence that this is the case. For example, O'Hara and Brown (40) analyzed the virulence of barley powdery mildew spores trapped over $10 \times 10 \mathrm{~m}$, isolated plots of host (barley) and nonhost (wheat) plants to estimate the proportion of spores derived from spore production within the plots as compared to immigration from outside the plots. Early in the season, the vast majority of trapped spores were immigrants. Once disease became established in the barley plots, however, the proportion of immigrants rapidly declined to near zero. This spatial independence is consistent with an earlier, conceptual view of Vanderplank (54), who proposed that polycyclic epidemics expand through monocyclic spread of colonizers at the epidemic front, followed by local, polycyclic build-up in the new area of colonization. It also is consistent with studies of wheat stripe rust indicating that focus size (C. C. Mundt, unpublished data) and host heterogeneity $(7,38)$ strongly influence the velocity of spread of wheat stripe rust, but host population size does not (47; C. C. Mundt, unpublished data).

\section{CONCLUSIONS}

Recent evidence suggests that the common assumption of random pathogen dispersal may be less adequate than previously expected, and that autoinfection may contribute a greater proportion of total infections than does alloinfection in at least some cases. Direct studies of autoinfection for additional diseases are needed, as the degree of autoinfection and mechanism by which it occurs are likely to be at least somewhat pathosystem-specific. For example, autoinfection apparently occurred via local aerial dispersal of urediniospores for wheat leaf rust (24), but by spread from leaf sheath infections to elongating stems and inflorescences for stem rust of ryegrass (42). Once measured, the epidemiological impacts of autoinfection must be accounted for, usually through modeling, with the appropriate approach depending on the question being asked. These approaches include modification of existing analytic models (e.g., modification of the carrying capacity term of the logistic equation), use of spatially explicit simulations that account for autoinfection and alloinfection, and meta-population approaches that attempt to relate dispersal patterns among different spatial scales. Given the substantial similarities between botanical and animal/human epidemiology (18, 29,38 ), autoinfection may be relevant to epizootics in many cases as well.

Effects of spatial scale have become of increasing interest in the fields of ecology (25) and epidemiology (30). Recent studies of autoinfection $(24,42,43,55,56)$ required painstaking, finescaled measurements not possible with coarse-grained sampling techniques commonly used in epidemiological studies. At the same time, however, the fine-scaled process of autoinfection has implications over a very wide range of spatial scale. The same may be true of temporal scale, as autoinfection is relevant to dispersal events occurring on the order of seconds to hours, but also to processes occurring on evolutionary time scales, such as local pathogen adaptation $(23,51)$ and evolution of virulence $(22,28)$.

\section{ACKNOWLEDGMENTS}

This work was supported by award 052756 from the NSF/NIH Ecology of Infectious Diseases Program.

\section{LITERATURE CITED}

1. Adee, S. R., Pfender, W. F., and Hartnett, D. C. 1990. Competition between Pyrenophora tritici-repentis and Septoria nodorum in the wheat leaf as measured with De Wit replacement series. Phytopathology 80:1177-1182.

2. Al-Naimi, F. A., Garrett, K. A., and Bockus, W. W. 2005. Competition, facilitation, and niche differentiation in two foliar pathogens. Oecologia 143:449-457.

3. Aylor, D. E., and Ferrandino, F. J. 1990. Initial spread of bean rust close to an inoculated bean leaf. Phytopathology 80:1469-1476.

4. Berger, R. D. 1981. Comparison of the Gompertz and logistic equations to describe plant disease progress. Phytopathology 71:716-719.

5. Burdon, J. J., and Chilvers, G. A. 1976. Controlled environment experiments on epidemics of barley mildew in different density host stands. Oecologia 26:61-72.

6. Cooke, B. M., Jones, D. G., and Kaye, B. (eds.) 2006. The Epidemiology of Plant Diseases. 2nd ed. Springer, Dordrecht, the Netherlands.

7. Cowger, C., Wallace, L. D., and Mundt, C. C. 2005. Velocity of spread of wheat stripe rust epidemics. Phytopathology 95:972-982.

8. da Luz, W. C., and Bergstrom, G. C. 1987. Interactions between Cochliobolus sativus and Pyrenophora tritici-repentis on wheat leaves. Phytopathology 77:355-1360.

9. Ferrandino, F. J. 1993. Dispersive epidemic waves: I. Focus expansion within a linear planting. Phytopathology 83:795-802.

10. Fleming, R. A. 1983. Development of a simple model of cereal rust progress. Phytopathology 73:308-312.

11. Fried, P. M., Mackenzie, D. R., and Nelson, R. R. 1979. Disease progress curves of Erysiphe graminis f. sp. tritici on Chancellor wheat and four multilines. Phytopathol. Z. 95:151-166. 
12. Garrett, K. A., and Mundt, C. C. 1999. Epidemiology in mixed host populations Phytopathology 89:984-900.

13. Gregory, L. V., Ayers, J. E., and Nelson, R. R. 1981. Reliability of apparent infection rates in epidemiological research. Phytopathol. Z. 100:135-142.

14. Gregory, P. H. 1968. Interpreting plant disease dispersal gradients. Annu. Rev. Phytopathol. 6:189-212.

15. Hastings, A., Cuddington, K., Davies, K. F., Dugaw, C. J., Elmendorf, S., Freestone, A., Harrison, S., Holland, M., Lambrinos, J., Malvadkar, U., Melbourne, B. A., Moore, K., Taylor, C., and Thomson, D. 2005. The spatial spread of invasions: New developments in theory and evidence. Ecol. Lett. 8:91-101.

16. Heesterbeek, J. A. P., and Zadoks, J. C. 1987. Modeling pandemics of quarantine pests and diseases: Problems and perspectives. Crop Prot. 6:211-221.

17. Imhoff, M. W., Leonard, K. J., and Main, C. E. 1982. Patterns of bean rust lesion size increase and spore production. Phytopathology 72:441-446.

18. Jeger, M. J. 2000. Theory and plant epidemiology. Plant Pathol. 49:651658.

19. Johnson, K. B. 1994. Dose-response relationships and inundative biological control. Phytopathology 84:780-784.

20. Katsuya, K., and Green, G. J. 1967. Reproductive potentials of races 15B and 56 of wheat stem rust. Can. J. Bot. 45:1077-1091.

21. Kinkel, L. L., and Lindow, S. E. 1997. Microbial competition and plant disease biocontrol. Pages 128-138 in: Ecological Interactions and Biological Control. D. Andow, D. Ragsdale, and R. Nyvall, eds. Westview Press, New York.

22. Kover, P. X., and Clay, K. 1998. Trade-off between virulence and vertical transmission and the maintenance of a virulent plant pathogen. Am. Nat. $152: 165-175$

23. Laine, A.-L. 2005. Spatial scale of local adaptation in a plant-pathogen metapopulation. J. Evol. Biol. 18:930-938.

24. Lannou, C., Soubeyrand, S., Frezal, L., and Chadoeuf, J. 2008. Autoinfection in wheat leaf rust epidemics. New Phytol. 177:1001-1011.

25. Leibold, M. A., Holyoak, M., Mouquet, N., Amarasekare, P., Chase, J. M., Hoopes, M. F., Holt, R. D., Shurin, J. B., Law, R., Tilman, D., Loreau, M., and Gonzalez, A. 2004. The metacommunity concept: A framework for multi-scale community ecology. Ecol. Lett. 7:601-613.

26. Leonard, K. J. 1968. Multiline varieties and oat stem rust population dynamics. Ph.D. thesis. Cornell University, Ithaca, NY.

27. Leonard, K. J. 1969. Factors affecting rates of stem rust increase in mixed plantings of susceptible and resistant oat varieties. Phytopathology 59:1845-1850.

28. Lipsitch, M., Siller, S., and Nowak, M. A. 1996. The evolution of virulence in pathogens with vertical and horizontal transmission. Evolution 50:1729-1741.

29. Madden, L. V., Hughes, G., and van den Bosch, F. 2007. The Study of Plant Disease Epidemics. The American Phytopathological Society, St. Paul, MN.

30. Matthews, L., and Haydon, D. 2007. Introduction. Cross-scale influences on epidemiological dynamics: From genes to ecosystems. J. Roy. Soc. Interf. 4:763-765.

31. Minogue, K. P., and Fry, W. E. 1983. Models for the spread of disease: Model description. Phytopathology 73:1168-1173.

32. Mundt, C. C. 1995. Models from plant pathology on the movement and fate of new genotypes of microorganisms in the environment. Annu. Rev. Phytopathol. 33:467-488.

33. Mundt, C. C. 2002. Use of multiline cultivars and cultivar mixtures for disease management. Annu. Rev. Phytopathol. 40:381-410.

34. Mundt, C. C., and Brophy, L. S. 1988. Influence of number of host genotype units on the effectiveness of host mixtures for disease control: A modeling approach. Phytopathology 78:1087-1094.

35. Mundt, C. C., and Browning, J. A. 1985. Development of crown rust epidemics in genetically diverse oat populations: Effect of genotype unit area. Phytopathology 75:607-610.
36. Mundt, C. C., and Leonard, K. J. 1985. A modification of Gregory's model for describing plant disease gradients. Phytopathology 75:930-935.

37. Mundt, C. C., Leonard, K. J., Thal, W. M., and Fulton, J. H. 1986. Computerized simulation of crown rust epidemics in mixtures of immune and susceptible oat plants with different genotype unit areas and spatial distributions of initial disease. Phytopathology 86:590-598.

38. Mundt, C. C., Sackett, K. E., Wallace, L. D., Cowger, C., and Dudley, J. P. 2009. Long distance dispersal and accelerating waves of disease: Empirical relationships. Am. Nat. 173:456-466.

39. Newton, M. R., Kinkel, L. L., and Leonard, K. J. 1997. Competition and density-dependent fitness in a plant parasitic fungus. Ecology 78:17741784.

40. O'Hara, R. B., and Brown, J. K. M. 1996. Immigration of the barley powdery mildew pathogen into field plots of barley. Plant Pathol. 45:1071-1076.

41. Parker, S. R., Shaw, M. W., and Royle, D. J. 1995. The reliability of visual estimates of disease severity on cereal leaves. Plant Pathol. 44:856-864.

42. Pfender, W. F. 2004. Role of phenology in host susceptibility and withinplant spread of stem rust during reproductive development of perennial ryegrass. Phytopathology 94:308-316.

43. Pfender, W. F. 2006. Interaction of fungicide physical modes of action and plant phenology in control of stem rust of perennial ryegrass grown for seed. Plant Dis. 90:1225-1232.

44. Plaut, J. L., and Berger, R. D. 1981. Infection rates in three pathosystem epidemics initiated with reduced disease severities. Phytopathology 71:917-921.

45. Robinson, R. A. 1976. Plant Pathosystems. Springer-Verlag, Berlin.

46. Rouse, D. I., MacKenzie, D. R., and Nelson, R. R. 1981. A relationship between initial inoculum and apparent infection rate in a set of disease progress data for powdery mildew on wheat. Phytopathol. Z. 100:143149.

47. Sackett, K. E., and Mundt, C. C. 2009. Effect of plot geometry on epidemic velocity of wheat yellow rust. Plant Pathol. 58:370-377.

48. Scherm, H. 1996. On the velocity of epidemic waves in model plant disease epidemics. Ecol. Mod. 87:217-222.

49. Skelsey, P., Rossing, W. A. H., Kessel, G. J. T, Powell, J., and van der Werf, W. 2005. Influence of host diversity on development of epidemics: An evaluation and elaboration of mixture theory. Phytopathology 95:328338

50. Tellier, A., and Brown, J. K. M. 2008. The relationship of host-mediated induced resistance to polymorphism in gene-for-gene relationships. Phytopathology 98:128-136.

51. Thrall, P. H., and Burdon, J. J. 2003. Evolution of virulence in a plant host-pathogen metapopulation. Science 299:1735-1737.

52. Van den Bosch, F., Zadoks, J. C., and Metz, J. A. J. 1988. Focus expansion in plant disease. I: The constant rate of focus expansion. Phytopathology 78:54-58.

53. Van der Plank, J. E. 1963. Plant Diseases: Epidemics and Control. Academic Press, New York.

54. Vanderplank, J. E. 1982. Host-Pathogen Interactions in Plant Disease. Academic Press, New York.

55. Vereijssen, J., Schneider, J. H. M., and Jeger, M. J. 2007. Epidemiology of Cercospora leaf spot on sugar beet: Modeling disease dynamics within and between individual plants. Phytopathology 97:1550-1557.

56. Willocquet, L., and Savary, S. 2004. An epidemiological simulation model with three scales of spatial hierarchy. Phytopathology 94:883-891.

57. Wilson, M., and Lindow, S. E. 1994. Coexistence among epiphytic bacterial populations mediated through nutritional resource partitioning. Appl. Environ. Microbiol. 60:4468-4477.

58. Xu, X.-M., and Ridout, M. S. 2000. Stochastic simulation of the spread of race-specific and race-nonspecific aerial fungal pathogens in cultivar mixtures. Plant Pathol. 49:207-218.

59. Zawolek, M. W., and Zadoks, J. C. 1992. Studies in focus development: An optimum for the dual dispersal of plant pathogens. Phytopathology 82:1299-1297. 